\title{
Loss of the Pioneer Factor FOXA1 Results in Genome-wide Epigenetic Reprogramming and activation
}

\section{of Interferon-Response Genes including CD274/PD-L1}

Wenhuo $\mathrm{Hu}^{1^{*}}$, Hironobu Yamashita ${ }^{2,3^{*}}$, Jenna Craig ${ }^{2,3}$, Vonn Walter ${ }^{4}$, Joshua I. Warrick ${ }^{2,3}$,

Hikmat Al-Ahmadie ${ }^{5}$, and David J. DeGraff',3,

1. Human Oncology and Pathogenesis Program, Memorial Sloan Kettering Cancer Center, New York, NY 10065

2. Department of Pathology and Laboratory Medicine, Penn State College of Medicine, Hershey, PA 17033

3. Department of Surgery, Division of Urology, Penn State College of Medicine, Hershey, PA 17033

4. Department of Public Health Sciences, Penn State College of Medicine, Hershey PA 17033

5. Department of Pathology, Memorial Sloan Kettering Cancer Center, New York, NY 10065

6. * indicates equal contribution

\section{Corresponding Authors and Requests for Reprints:}

David J. DeGraff, Ph.D.

Pennsylvania State University College of Medicine

Department of Pathology and Laboratory Medicine

Department of Surgery, Division of Urology

500 University Drive

Hershey PA 17033-0850

717-531-0003; Extension 281295
Hikmat Al-Ahmadie, M.D.

Department of Pathology

Memorial Sloan Kettering Cancer Center

1275 York Avenue

New York, NY 10065

212-639-5905

alahmadh@mskcc.org 


\section{Abstract}

Forkhead Box A1 (FOXA1) is a pioneer transcription factor critical in epigenetic regulation of chromatin and cell fate determination. Reduced FOXA1 expression is an independent predictor of poor overall survival in bladder cancer patients. However, the impact of FOXA1 loss on chromatin epigenetics in bladder cancer is unknown. Therefore, we determined the impact of FOXA1 knock out (KO) on epigenetic modification of chromatin and associated gene expression. We identified 8,230 differentially expressed genes following FOXA1 KO. Surprisingly, Gene Set Enrichment Analysis (GSEA) identified IFNa/y gene expression signatures as enriched following FOXA1 KO. FOXA1 KO induced both increased and decreased numbers of histone 3 lysine 27 acetylation (H3K27ac) sites throughout the genome. As expected, the majority of differences in H3K27ac across genomic areas in FOXA1 KO cells is mapped to intergenic and intronic regions where enhancers reside. In addition, a subset of differential H3K27ac levels were also mapped to proximal promoters and within gene bodies. Integrated analysis of RNA/ChIP-seq data shows changes in gene expression that are mirrored by differences in H3K27ac. Motif analysis of DNA sequence enriched for H3K27ac identified significant increases in transcription factor binding motifs including the interferon sensitive response element (ISRE) and interferon response factors such as IRF1. Moreover, we identified increased H3K27ac of regulatory elements as being associated with several upregulated interferon sensitive genes (ISGs) in FOXA1 KO cells, including CD274/PDL1. Western blotting and Q-RT-PCR confirmed upregulation of CD274/PD-L1 following FOXA1 KO. Analysis of TCGA data confirmed an inverse relationship between FOXA1 and CD274 in bladder cancer, as well as in other cancers. In summary, we provide evidence of widespread epigenetic reprogramming after FOXA1 KO in bladder cancer cells. Additionally, we provide evidence that FOXA1 KO-induced epigenetic changes contribute to activation of a global interferon-dominant expression signature, including the immune checkpoint target CD274/PD-L1 in a cancer cell-intrinsic manner. 


\section{Introduction}

We identified loss of Forkhead Box A1 (FOXA1) expression as a common event in the development of bladder cancers with squamous differentiation ${ }^{1}$, and subsequently showed that FOXA1 is an independent predictor of poor overall survival in this common malignancy ${ }^{2}$. Additional in vitro studies showed that FOXA1 overexpression cooperates with overexpression of GATA Binding Protein 3 (GATA3) and pharmacologic activation of Peroxisome Proliferator Activated Receptor Gamma (PPARy) to repress a basal-squamous signature in vitro ${ }^{3}$. Moreover, we went on to show that in vivo ablation of FOXA1 results in the development of squamous differentiation and cooperates with other genetic events resulting in the development of bladder cancer ${ }^{4}$. While our observations have identified an important role for this pioneer factor in bladder cancer, the mechanism by which FOXA1 impacts gene expression in bladder cancer remains unknown.

In addition to performing a role in organogenesis and tissue-specific gene expression, pioneer transcription factors such as FOXA1 directly bind to areas of condensed chromatin prior to recruitment of other coregulatory factors ${ }^{5,6}$. Indeed, FOXA1-chromatin binding plays an important biologic role for organogenesis and tissue-specific gene expression ${ }^{7}$. Because of the structural similarity of the FOXA1 DNA binding domain to linker histone ${ }^{8}$, FOXA1 can displace linker histone, resulting in increases in local DNA accessibility to enable binding of additional trans acting factors ${ }^{9}$. In addition, FOXA1 binding to chromatin can result in the "passive" reduction in the number of additional binding events required for transcriptional activation ${ }^{9}$. Moreover, bookmarking of specific genomic loci by FOXA1 during mitosis enables rapid expression of genes following mitotic exit, thus preserving cell identify ${ }^{10}$. Interestingly, active and passive pioneering, as well as bookmarking by FOXA1 are accompanied by epigenetic changes in chromatin modification, which are in turn associated with DNA accessibility by trans-acting factors, as well as gene expression changes.

In addition to a role in organogenesis and tissue-specific gene expression, ample evidence indicates a role for FOXA1-mediated regulation of chromatin accessibility in disease pathogenesis. For example, alterations in FOXA1 expression or mutational status have been associated with chromatin alterations in hormonally-regulated cancers of the breast and prostate ${ }^{11-14}$, as well as other malignancies including liver ${ }^{15}$ and pancreatic cancer ${ }^{16}$. Therefore, we hypothesized that decreased expression of FOXA1 in urothelial cancer cells has an important, and disease-relevant impact on epigenetic post-translational modification of chromatin. 


\section{Results}

FOXA1 is a cancer cell-intrinsic repressor of the IFNa and IFNy transcriptional signature including CD274/PD-L1 expression.




To gain insight into alterations in gene expression associated with FOXA1 inactivation, we genetically ablated FOXA1 in luminal UMUC1 bladder cancer cells, resulting in the creation of two separate knockout (KO) clones. Principal component analysis performed on RNA seq data identified a clear separation between parental and FOXA1 KO cells (Figure 1A). Differential expression analysis identified 8,230 differentially expressed genes following FOXA1 KO (Figure 1B; 4,185 increased, 4,045 decreased; FDR q<0.05; two separate KO clones, average of differentially expressed genes in both clones). Interrogation of upregulated genes from individual UMUC1 FOXA1 KO clones via Gene Set Enrichment Analysis (GSEA) analysis strongly suggested a role for FOXA1 in repression of interferon target genes (Figure 1C). For example, GSEA identified both "interferon alpha (IFNa) response" (FOXA1 KO clone 1; GSEA pre-rank analysis FDR q = 0.005, NES = 1.79; FOXA1 KO clone 2, GSEA pre-rank analysis FDR $q<0.001, \mathrm{NES}=2.54)$ and "interferon gamma $(\mathrm{IFN \gamma})$ response" $(F O X A 1 \mathrm{KO}$ clone 1; GSEA pre-rank analysis FDR $\mathrm{q}=0.04$, NES $=1.42 ;$ FOXA1 KO clone 2, GSEA pre-rank analysis FDR $q<0.001, \mathrm{NES}=2.02$ ) as being enriched following FOXA1 ablation (Figure 1C). Amongst genes expressed at significantly lower levels following FOXA1 KO, enriched gene sets included "MYC targets V1", "E2F targets" and "androgen response" (data not shown). This latter finding is noteworthy because of the fact that FOXA1 is known to interact with the androgen receptor to dictate the androgen responsive cistrome ${ }^{17-19}$. We next focused the expression analysis on genes relevant to interferon signaling, and found FOXA1 KO cells demonstrated significantly increased expression of $C D 274$, the gene encoding the immune checkpoint target Programmed Death-Ligand 1 (Figure 1D; $\mathrm{q}<0.05$ for both FOXA1 KO clone 1 and 2). This is in keeping with the fact that FOXA1 has been previously identified as a direct, positive regulator of CD274PD-L1 in a subset of T regulatory cells $^{20}$. In addition, FOXA1 KO resulted in significantly increased expression of several other interferon-sensitive genes (ISGs), including IFI35, IRF9, IFIT2 and STAT2 among others (Figure 1D; $\mathrm{q}<0.05$ for both FOXA1 KO clone 1 and 2).

Based on these results, we further investigated the impact of altered FOXA1 levels on CD274/PD-L1 expression. Q-RT-PCR (Figure 2A and B; Student's t-test; $p<0.05$ ) and western blotting (Figure 2C, D and E; Student's t-test; $\mathrm{p}<0.05)$ of parental UMUC1 and UMUC1 FOXA1 KO cells confirmed significantly increased expression of CD274/PD-L1 following FOXA1 KO. The ability of FOXA1 to regulate CD274/PD-L1 was additionally supported by FOXA1 gain of function studies. Specifically, we identified significant decreases in CD274/PD-L1 expression following FOXA1 overexpression in UMUC3 bladder cancer cells (Figure. 2F thru J; 
Student's t-test; $p<0.05)$. Analysis of publicly available data from TCGA confirmed an inverse relationship between FOXA1 and CD274 expression in a cohort of bladder cancer patients (Figure 2K; Spearman correlation; $r=-0.45 ; p<0.001)$. In addition to bladder cancer, analysis of pan-cancer TCGA data identified a statistically significant associations between FOXA1 and CD274 expression in a number of malignancies (Figure 2L). In summary, these findings identify FOXA1 as a cancer cell-intrinsic repressor of CD274/PD-L1 in bladder cancer, as well as potentially in other malignancies.

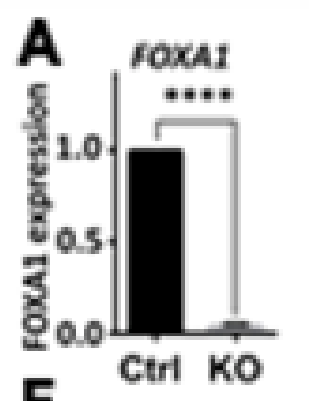

$\mathbf{F}$

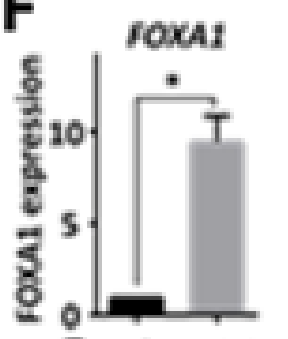

EmptyFoxA1
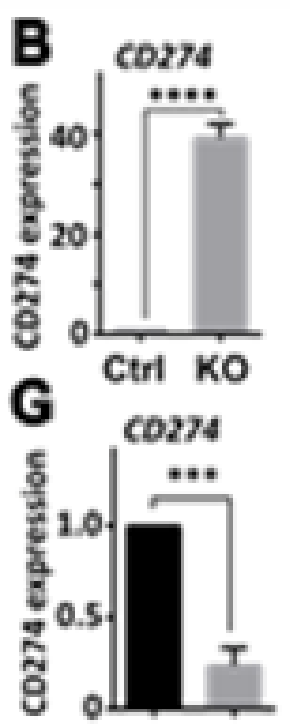

Empty FOXA1
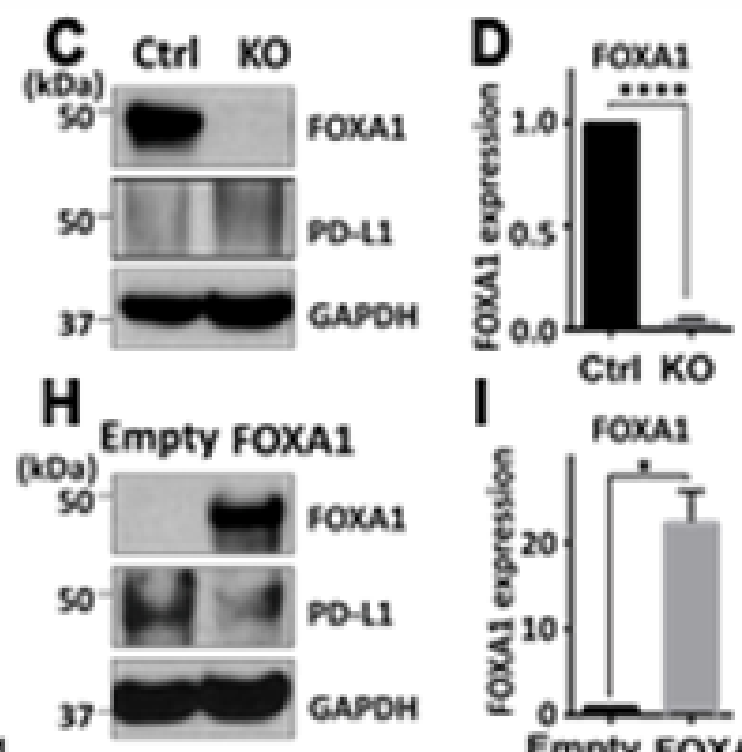

Empty FOXA1
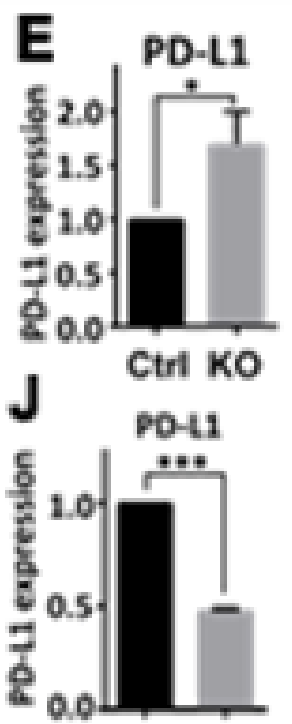

Empty FOXA1


Figure 2: $C D 274 /$ PD-L1 is a FOXA1-repressed immune checkpoint target. Q-RT-PCR (A and B), western blotting $(\mathbf{C})$ and densitometry (D and E) confirms decreased FOXA1 and increased CD274/PD-L1 expression in UMUC1 FOXA1 KO cells. Q-RT-PCR (F and $\mathbf{G})$, western blotting $(\mathbf{H})$ and densitometry (I and J) confirms increased FOXA1 and decreased CD274/PD-L1 expression in UMUC3 FOXA1 overexpressing cells. (K) Inverse correlation between FOXA1 and CD274 (encoding PD-L1 expression) in the TCGA bladder data set $(\mathrm{r}=-.45 ; \mathrm{p}<0.001$; Spearman correlation test). (L) Spearman correlation of FOXA1 and CD274 across cancers identifies a significant correlation between these genes in a number of malignancies. 


\section{Activation of the interferon-dominant immune signature and CD274/PD-L1 is a result of genome-wide epigenetic reprogramming following FOXA1 ablation.}

The ability of FOXA1 to regulate chromatin accessibility at enhancer and promoter elements via displacement of linker histone is a hallmark characteristic of this and other pioneer factors ${ }^{5,9}$. Genome-wide changes in one mark of active enhancers and promoters, Histone 3 lysine 27 acetylation (H3K27ac), has been reported following changes in FOXA1 expression in a number of malignancies ${ }^{16,21}$. While the impact of FOXA1 silencing on the epigenomic landscape in bladder cancer has not been reported, we hypothesized that increased expression of CD274 and other ISGs may be a result of changes in H3K27ac of associated genes following FOXA1 loss. To directly test this hypothesis, we subjected parental and UMUC1 FOXA1 KO (clone 2; two technical replicates) to chromatin immunoprecipitation coupled to high throughput sequencing (ChIP-seq) for H3K27ac, and integrated these results with our RNA-seq data following FOXA1 KO. There were 14,977 locus with H3K27ac enrichment in either parental UM-UC-1 cells or its FOXA1 KO cells (FDR < 0.05). Consistent with the accepted role of FOXA1 in the control of enhancer activity, the majority of differences in H3K27ac areas following FOXA1 KO were mapped to intergenic ( $n=6,250$ peaks; $42 \%$ of total peaks) and intronic $(n=6,490$ peaks; 43\%) genomic regions (Figure 3A). In addition, differential H3K27ac levels following FOXA1 KO were also mapped to proximal promoters ( $n=1,306$ peaks; $9 \%$ ) as well as within gene bodies (931 peaks; $6 \%$ ).

Within the 6,250 H3K27ac peaks enriched in intergenic region, we identified 4 distinct clusters (Figure 3B). Clusters 1 ( $n=943$ peaks) and 3 ( $n=2499$ peaks) exhibited significant increases in H3K27ac levels, while clusters 2 ( $n=883$ peaks) and 4 ( $n=1925$ peaks) exhibited significant decreases in H3K27ac marks at intergenic loci. Confirming an important role for FOXA1 in the regulation of enhancer activity, integrated RNA-seq and ChIP-seq results identified significant changes in gene expression mirrored differences in H3K27ac (Figure 3B). While intergenic regions showed both significant numbers of decreased and increased H3K27ac marks following FOXA1 KO, we identified widespread increases in H3K27ac marks at proximal promoters (Figure 3C). Specifically, 3 separate clusters were identified, with clusters 1 ( $n=484$ peaks; 37\% of promoter peaks) and 2 ( $n=664$ peaks; $51 \%$ of promoter peaks) exhibiting increases in H3K27ac marks, while cluster 3 exhibited a 
A

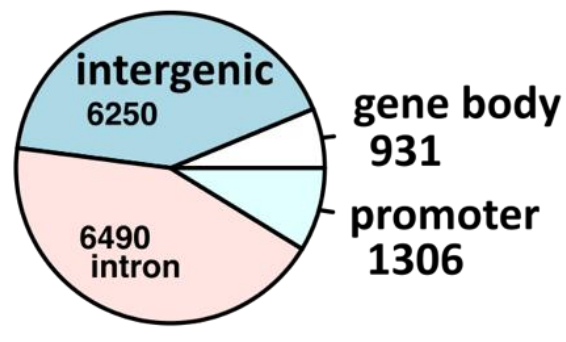

B

H3K27ac ChIP-Seq

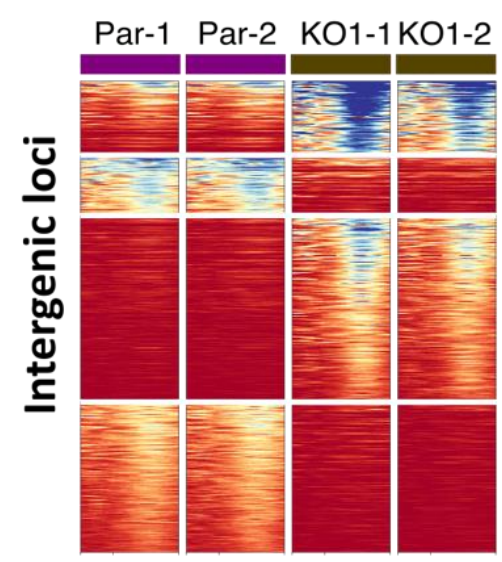

\section{C}

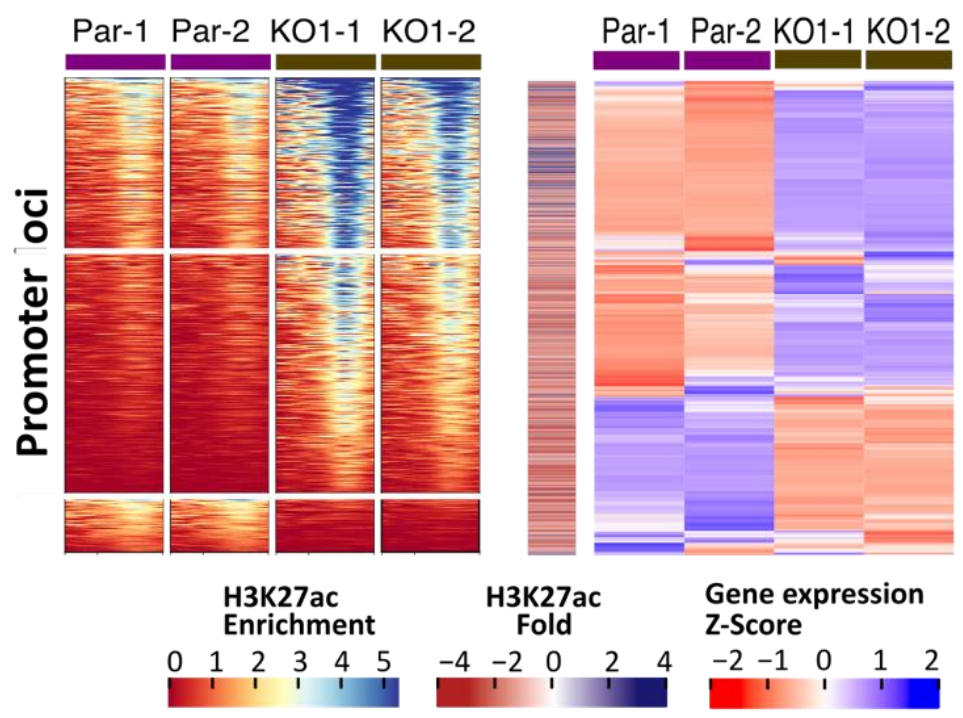

D

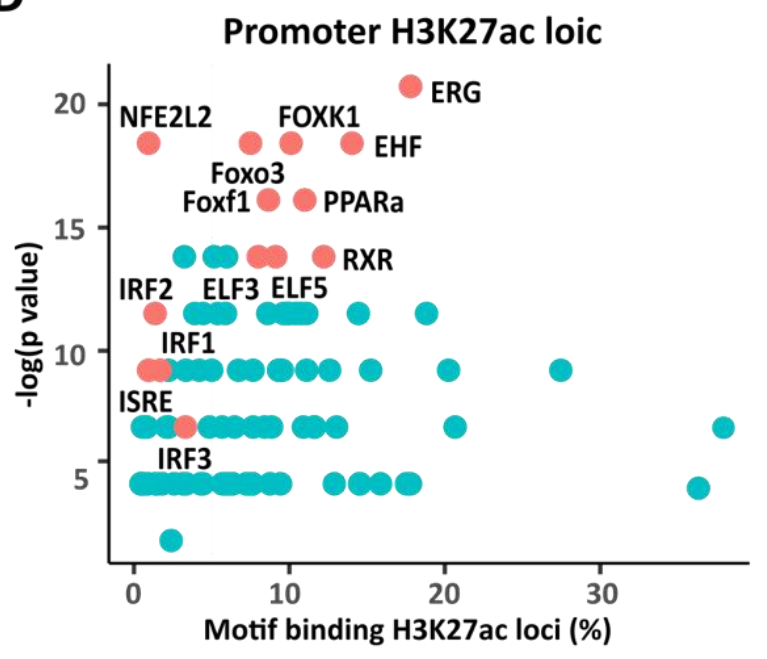

E

ISRE motif

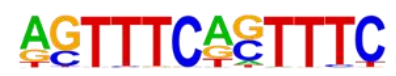

-170: TGTTTCACTTTC
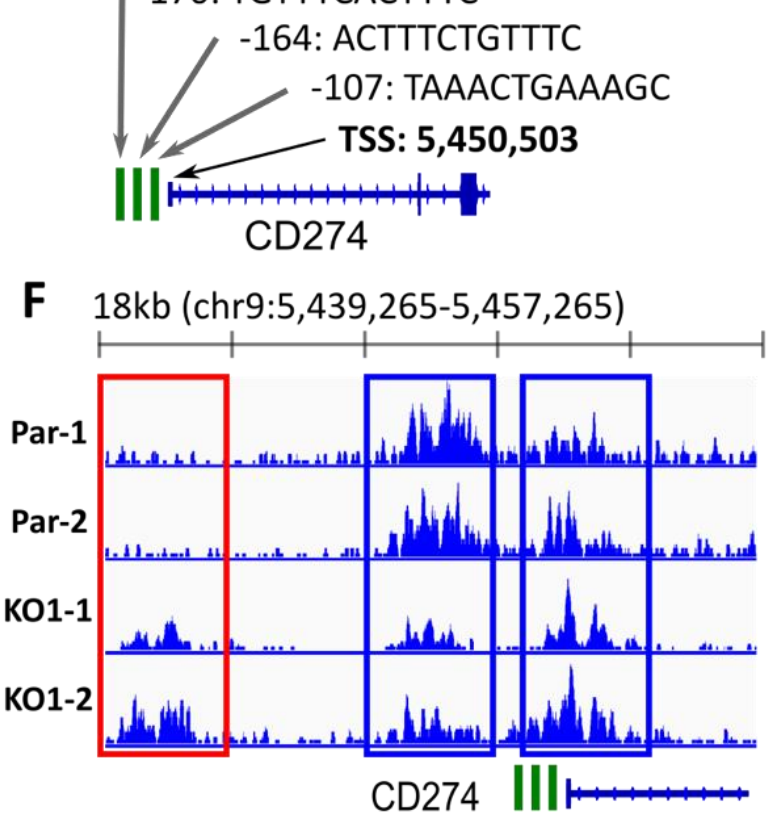

Figure 3: Genetic knockout of $F O X A 1$ results in widespread enhancer and promoter reprogramming in human cells (A) Genomic region annotation of H3K27ac modification locus. (B and C) H3K27ac cluster analysis (left panels) and expression heatmaps (right panels) showing relationship between H3K27ac ChIPseq and expression of 1,724 associated genes (RNA-seq) at (B) intergenic (including enhancers) and (C) promoter levels. These data show decreased $\mathrm{H} 3 \mathrm{~K} 27 \mathrm{ac}$ is associated with reduced gene expression following FOXA1 KO, while increased $\mathrm{H} 3 \mathrm{~K} 27$ ac is associated with increased gene expression following FOXA1 KO. (D) Motif analysis identifying enrichment of binding sites for transcription factors activated by IFN $\alpha / \gamma$ signaling at areas of increased H3K27ac following FOXA1 KO. (E) Illustration identifying the location of several interferon-sensitive response element (ISRE) motifs in the areas of increased acetylation in the $C D 274$ promoter. (F) Increased acetylation of $C D 274$ regulatory elements including an upstream enhancer (red box) and the proximal promoter region (blue boxes) following FOXA1 KO. 
reduction in H3K27ac marks ( $n=158$ peaks; $12 \%$ of promoter peaks). In keeping with increased levels of H3K27ac at promoters, expression of greater than half of genes in clusters 1 and 2 were upregulated following RNA-seq analysis (Figure 3C). These results suggest an important, yet perhaps underappreciated role for FOXA1 in the control of proximal promoter activity. As H3K27ac marks areas of open chromatin associated with active regulatory elements ${ }^{22,23}$, we performed motif analysis in an effort to determine what transcription factors potentially bind to newly accessible areas following FOXA1 KO. In support of a role for FOXA1 KO in the global activation of ISGs, enriched transcription factor binding motifs included the interferon sensitive response element (ISRE; q<0.05), as well as motifs for interferon response factors (IRF1, IRF2 and IRF3; all q<0.05) (Figure 3D). Focusing on the CD274 proximal promoter, we identified 3 separate ISRE elements in this region (Figure 3E). As predicted by our motif analysis, these three ISRE elements overlapped with areas of H3K27ac following in both parental and FOXA1 KO UMUC1 cells, which suggests basal promoter activity (Figure 3F). In addition, we identified an additional regulatory element upstream of CD274 as having increased H3K27ac following FOXA1 KO (Figure 3F). In summary, we provide evidence of widespread epigenetic reprogramming following FOXA1 KO in bladder cancer cells. Additionally, we provide evidence that epigenetic changes contribute to activation of a global interferon-dominant signature, including CD274/PD-L1 in a cancer cell-intrinsic manner following FOXA1 inactivation.

\section{Discussion}

This short report identifies FOXA1 as a repressor of CD274/PD-L1 expression, indicating a tumor cellintrinsic contribution to PD-L1 expression. Increased H3K27ac is an epigenomic mark of increased activity of cis regulatory elements ${ }^{22,}{ }^{23}$. We found that FOXA1 inactivation in vitro drives genome-wide epigenetic reprogramming via alterations in chromatin acetylation, specifically increasing H3K27ac at regulatory elements important for the control of CD274/PD-L1 and other ISGs. The identification of FOXA1 as a tumor cell-intrinsic repressor of CD274/PD-L1 expression was further supported by gain of function studies. Mutations in FOXA1 have been associated with activation of IFNy-target genes in prostate cancer ${ }^{12}$, and FOXA1 has previously been implicated in the positive control of PD-L1 expression in a subset of immune cells ${ }^{20}$. However, to the best of our knowledge this is the first report of FOXA1 as a tumor cell-intrinsic repressor of PD-L1 in any malignancy. FOXA1 is a recognized pioneer factor controlling chromatin structure ${ }^{5,6}$, and our findings suggest global epigenetic reprogramming following FOXA1 loss contributes to the activation of IFN-dominant signatures. This suggestion 
is supported by motif analysis following H3K27ac ChIP-seq, which demonstrated that areas of acetylated DNA following FOXA1 loss are enriched for binding sites of a number of interferon-responsive factors. This finding suggests that FOXA1 inactivation "primes" tumor cells, making them more sensitive to paracrine interferons produced by infiltrating inflammatory cells. Furthermore, the possibility that FOXA1 serves as an important regulator of PD-L1 in other malignancies is supported by our retrospective analysis of TCGA data. More work is required to determine the functional implications increased ISGs and PD-L1 expression for infiltrating immune cells.

\section{Materials and Methods}

\section{Cell culture and generation of FOXA1 knockout lines}

Human bladder cancer cells (UMUC1, UMUC3) were purchased as described previously ${ }^{3}$, and authenticity was confirmed by short tandem repeat (STR) analysis. Cells were cultured in Minimal Essential Medium (UMUC1, UMUC3) supplemented with 10\% FBS. To establish UMUC1-FOXA1 knockout (KO), UMUC1 (2x105) cells were transfected with $2.5 \mathrm{mg}$ of HNF-3alpha CRISPR/Cas9 KO plasmid (Santa Cruz, sc-400743) using lipofectamine3000 (Thermo fisher scientific). After $48 \mathrm{~h}$, transfected cells were trypsinized and resuspended in PBS. Three GFP-positive cells were FACs sorted in single well of 96-well plate containing 100 ml of medium. Sorted cells were expanded and sequentially transferred to 24 well, 6 well dishes, and T-75 flasks. Finally, knockout of FOXA1 in UMUC1-FOXA1KO cells was confirmed by qPCR and western blot analysis.

\section{ChIP-Seq}

Cells were fixed with $1 \%$ formaldehyde for $15 \mathrm{~min}$ and quenched with $0.125 \mathrm{M}$ glycine. Chromatin was isolated by the addition of lysis buffer, followed by disruption with a Dounce homogenizer. Lysates were sonicated, and the DNA sheared to an average length of 300-500 bp. Genomic DNA (Input) was prepared by treating aliquots of chromatin with RNase, proteinase $\mathrm{K}$ and heat for de-crosslinking, followed by ethanol precipitation. Pellets were resuspended, and the resulting DNA was quantified on a NanoDrop spectrophotometer. Extrapolation to the original chromatin volume allowed quantitation of the total chromatin yield. An aliquot of chromatin (30 ug) was precleared with protein A agarose beads (Invitrogen). Genomic DNA regions of interest were isolated using 4 ug of antibody against H3K27Ac (Active Motif, cat\# 39133, Lot\# 28518012). Complexes were washed, eluted 
from the beads with SDS buffer, and subjected to RNase and proteinase $\mathrm{K}$ treatment. Crosslinks were reversed by incubation overnight at $65 \mathrm{C}$, and ChIP DNA was purified by phenol-chloroform extraction and ethanol precipitation. Quantitative PCR (QPCR) reactions were carried out in triplicate on specific genomic regions using SYBR Green Supermix (Bio-Rad). The resulting signals were normalized for primer efficiency by carrying out QPCR for each primer pair using Input DNA. Illumina sequencing libraries were prepared from the ChIP and Input DNAs by the standard consecutive enzymatic steps of end-polishing, dA-addition, and adaptor ligation. Steps were performed on an automated system (Apollo 342, Wafergen Biosystems/Takara). After a final PCR amplification step, the resulting DNA libraries were quantified and sequenced on Illumina's NextSeq 500 (75 nt reads, single end). Reads were aligned to the human genome (hg38) using the Bowtie2 algorithm (default settings). Duplicate reads were removed and only uniquely mapped reads (mapping quality $>=25$ ) were used for further analysis (average deduplicated 23 millions of reads per sample). Peak locations were determined using the MACS algorithm (v2.1.0) with a cutoff of $p$-value = 1e-7. Peaks that were on the ENCODE blacklist of known false ChIP-Seq peaks were removed. Differentially modified locus of H3K27ac locus were identified by DiffBind package (v2.16). Peak annotation was performed by Homer program (v4.11). Promoter region ciselement prediction was performed by universalmotif package (v1.6.3).

RNA extraction, quantitative real time $P C R(q R T-P C R)$ and western blotting analysis

Total RNA was extracted using the RNeasy kit (Qiagen) according to the manufacturer's protocol. For cDNA synthesis, reverse transcription was performed using M-MLV reverse transcriptase (Thermo Fisher Scientific) via manufacturer instructions. qRT-PCR was performed using QuantaStudio7 Real-Time PCR System (Applied Biosystems). Taqman probes used in this study were as follows. FOXA1 (Hs04187555_m1), CD274 (Hs00204257_m1). Relative gene expression change was calculated by the deltadeltaCt method. 18S ribosomal RNA was used as an endogenous reference. Western blotting was performed as described previously ${ }^{3}$. Primary antibodies were used as follows. FOXA1 (1:500, ab23738, Abcam), PD-L1 (1:1000, ab213524, abcam), GAPDH (14C10) (1:1000, \#2118, Cell signaling). 
Briefly, RNA was extracted using RNeasy mini kit (Qiagen; Valencia, CA) according to manufacturer's instructions. After ribogreen quantification and quality control with an Agilent BioAnalyzer, 2ug of total RNA underwent polyA selection and Truseq library (TruSeq ${ }^{\mathrm{TM}}$ RNA Sample Prep Kit v2) preparation. Briefly, samples were fragmented for 2 minutes at 94C before undergoing first strand and second strand cDNA synthesis. Libraries were amplified with 10 cycles of PCR and size-selected for fragments between 400 and 550 bp with a Pippin prep instrument (Sage Science). Samples were barcoded and run on a Hiseq 2500 in a $100 \mathrm{bp}$ paired end run, using the TruSeq SBS Kit v3 (Illumina). Sequence reads were aligned and counted for each gene by RSEM algorithm (26) with the STAR alignment program. An average of 30 million paired reads were achieved per sample, reads normalization and gene expression were analyzed using DESeq2. Gene pathway analysis was performed by GSEA.

\section{Acknowledgments}

Supported in part by RSG 17-233-01-TBE from the American Cancer Society (D.J.D.), The W.W. Smith Charitable Trust (D.J.D.), the Ruth Heisey Cagnoli Endowment in Urology at Penn State College of Medicine, the Bladder Cancer Support Group at Penn State Health, the Leo and Anne Albert Institute for Bladder Cancer Care \& Research, the Sloan Kettering Institute for Cancer Research Cancer Center Support Grant (P30CA008748), the SPORE in Bladder Cancer (P50CA221745) and R01CA233899 (H.A.).

\section{References}

1. DeGraff DJ, Clark PE, Cates JM, et al. Loss of the urothelial differentiation marker FOXA1 is associated with high grade, late stage bladder cancer and increased tumor proliferation. PLoS One. 2012;7(5):e36669. doi:10.1371/journal.pone.0036669

2. Reddy OL, Cates JM, Gellert LL, et al. Loss of FOXA1 Drives Sexually Dimorphic Changes in Urothelial Differentiation and Is an Independent Predictor of Poor Prognosis in Bladder Cancer. Am J Pathol. May 2015;185(5):1385-95. doi:10.1016/j.ajpath.2015.01.014

S0002-9440(15)00083-8 [pii]

3. Warrick JI, Walter V, Yamashita H, et al. FOXA1, GATA3 and PPARy Cooperate to Drive Luminal Subtype in Bladder Cancer: A Molecular Analysis of Established Human Cell Lines. Sci Rep. Dec 2016;6:38531. doi:10.1038/srep38531

4. Osei-Amponsa V, Buckwalter JM, Shuman L, et al. Hypermethylation of FOXA1 and allelic loss of PTEN drive squamous differentiation and promote heterogeneity in bladder cancer. Oncogene. Oct 2019;doi:10.1038/s41388-0191063-4

5. Cirillo LA, Lin FR, Cuesta I, Friedman D, Jarnik M, Zaret KS. Opening of compacted chromatin by early developmental transcription factors HNF3 (FoxA) and GATA-4. Mol Cell. Feb 2002;9(2):279-89. doi:10.1016/s10972765(02)00459-8 
6. Iwafuchi-Doi M, Donahue G, Kakumanu A, et al. The Pioneer Transcription Factor FoxA Maintains an Accessible Nucleosome Configuration at Enhancers for Tissue-Specific Gene Activation. Mol Cell. Apr 2016;62(1):79-91.

doi:10.1016/j.molcel.2016.03.001

7. Kaestner KH. The FoxA factors in organogenesis and differentiation. Curr Opin Genet Dev. Oct 2010;20(5):527-

32. doi:10.1016/j.gde.2010.06.005

8. Zaret KS, Caravaca JM, Tulin A, Sekiya T. Nuclear mobility and mitotic chromosome binding: similarities between pioneer transcription factor FoxA and linker histone H1. Cold Spring Harb Symp Quant Biol. 2010;75:219-26. doi:10.1101/sqb.2010.75.061

9. Zaret KS, Carroll JS. Pioneer transcription factors: establishing competence for gene expression. Genes Dev. Nov 2011;25(21):2227-41. doi:10.1101/gad.176826.111

10. Palozola KC, Lerner J, Zaret KS. A changing paradigm of transcriptional memory propagation through mitosis. Nat Rev Mol Cell Biol. 01 2019;20(1):55-64. doi:10.1038/s41580-018-0077-z

11. Lupien M, Eeckhoute J, Meyer CA, et al. FoxA1 translates epigenetic signatures into enhancer-driven lineagespecific transcription. Cell. Mar 2008;132(6):958-70. doi:10.1016/j.cell.2008.01.018

12. Xu B, Song B, Lu X, et al. Altered chromatin recruitment by FOXA1 mutations promotes androgen independence and prostate cancer progression. Cell Res. Sep 2019;29(9):773-775. doi:10.1038/s41422-019-0204-1

13. Parolia A, Cieslik M, Chu SC, et al. Distinct structural classes of activating FOXA1 alterations in advanced prostate cancer. Nature. 07 2019;571(7765):413-418. doi:10.1038/s41586-019-1347-4

14. Adams EJ, Karthaus WR, Hoover E, et al. FOXA1 mutations alter pioneering activity, differentiation and prostate cancer phenotypes. Nature. 07 2019;571(7765):408-412. doi:10.1038/s41586-019-1318-9

15. Li Z, Tuteja G, Schug J, Kaestner KH. Foxa1 and Foxa2 are essential for sexual dimorphism in liver cancer. Cell. Jan 2012;148(1-2):72-83. doi:10.1016/j.cell.2011.11.026

16. Roe JS, Hwang Cl, Somerville TDD, et al. Enhancer Reprogramming Promotes Pancreatic Cancer Metastasis. Cell. Aug 2017;170(5):875-888.e20. doi:10.1016/j.cell.2017.07.007

17. Wang $\mathrm{Q}, \mathrm{Li} \mathrm{W}$, Zhang $\mathrm{Y}$, et al. Androgen receptor regulates a distinct transcription program in androgenindependent prostate cancer. Cell. Jul 2009;138(2):245-56. doi:10.1016/j.cell.2009.04.056

18. Gao N, Zhang J, Rao MA, et al. The role of hepatocyte nuclear factor-3 alpha (Forkhead Box A1) and androgen receptor in transcriptional regulation of prostatic genes. Mol Endocrinol. Aug 2003;17(8):1484-507.

doi:10.1210/me.2003-0020

19. Gao N, Ishii K, Mirosevich J, et al. Forkhead box A1 regulates prostate ductal morphogenesis and promotes epithelial cell maturation. Development. Aug 2005;132(15):3431-43. doi:10.1242/dev.01917

20. Liu Y, Carlsson R, Comabella M, et al. FoxA1 directs the lineage and immunosuppressive properties of a novel regulatory T cell population in EAE and MS. Nat Med. Mar 2014;20(3):272-82. doi:10.1038/nm.3485

21. Fu X, Pereira R, De Angelis C, et al. FOXA1 upregulation promotes enhancer and transcriptional reprogramming in endocrine-resistant breast cancer. Proc Natl Acad Sci U S A. Dec 2019;doi:10.1073/pnas.1911584116

22. Kouzarides T. Chromatin modifications and their function. Cell. Feb 2007;128(4):693-705.

doi:10.1016/j.cell.2007.02.005

23. Creyghton MP, Cheng AW, Welstead GG, et al. Histone H3K27ac separates active from poised enhancers and predicts developmental state. Proc Natl Acad Sci U S A. Dec 2010;107(50):21931-6. doi:10.1073/pnas.1016071107 